\title{
Spoken and Typed Expressions of Repeated Attitudes: Matching Response Modes Leads to Attitude Retrieval versus Construction
}

\author{
NADER T. TAVASSOLI \\ GAVAN J. FITZSIMONS*
}

\begin{abstract}
Speaking and typing recruit different cognitive, motor, and perceptual systems that result in the encoding of differentiated memory traces. These factors did not affect the expression of stimulus-based attitudes. However, matching response modes resulted in more consistent repeated attitudes in experiment 1 and more predictable choice behaviors in experiment 2 than mismatching response modes. Judgmentconfidence and recall data in experiment 3 indicate that matching (mismatching) response modes leads to attitude retrieval (construction). These findings are of growing relevance to marketers and opinion pollsters who assess attitudes expressed orally and, increasingly, in typed form over the Internet.
\end{abstract}

$\mathrm{C}$ onsider a situation in which a consumer expresses an attitude in an Internet chat room. A second consumer expresses an attitude in a face-to-face conversation. At a later point in time both again express their attitudes in a telephone conversation. Whose attitudes will be more consistent across the two occasions? Are their delayed attitudes simply retrieved, or are they reconstructed? What if each of the consumers had instead typed the second attitude in an online review? These questions are not only of theoretical interest but also of practical importance for marketers and opinion pollsters who often repeatedly measure attitudes in order to track attitude change or to assess the impact of a marketing intervention. Previous consumer and psychological research is mute with respect to these questions and tacitly assumes that the essential elements that differentiate oral and manual responses compose motor components alone. This article questions this assumption.

We do not examine initial attitudes that are formed without being expressed. Nor do we expect initial stimulus-based evaluations to depend on the response mode. In many sit-

\footnotetext{
*Nader Tavassoli is professor of marketing at London Business School, London NW1 4SA, UK (ntavassoli@london.edu). Gavan Fitzsimons is associate professor of marketing and psychology at the Fuqua School of Business, Duke University, Box 90120, 1 Towerview Drive, Durham, NC 27708 (gavan@duke.edu). Correspondence: Nader Tavassoli or Gavan Fitzsimons. The authors gratefully acknowledge funding by the Centre for Marketing at London Business School and insightful comments by John Lynch, three reviewers, and the editors.
}

Dawn Iacobucci served as editor and Gita Johar served as associate editor for this article.

Electronically published July 28, 2006 uations, however, judgments and choices are memory based and influenced by past judgments an individual has expressed (Lingle and Ostrom 1979). It is in this context, for the repeated expression of attitudes, that we question the assumption that attitudes are neutral with respect to response mode. In three experiments, we find that delayed attitudes and choices are more consistent with initial attitudes when expressed in the same response mode. In the third experiment we provide evidence that these differences result from different cognitive processes: initial attitudes appear more likely to be retrieved when the response mode of the delayed attitude is the same, whereas delayed attitudes appear more likely to be constructed based on learned product information when expressed in a different response mode from the initial attitude.

\section{ATTITUDE RETRIEVAL VERSUS CONSTRUCTION}

The degree of agreement between initial and delayed attitudes is an important concern for both theorists and practitioners interested in persuasion effects. Research has found that the factors driving the valence and extremity of an initial evaluation may be very different from the factors determining consistency (Cook and Flay 1978). Research has also found that the degree of correspondence between initial and delayed attitudes is often low and that attitudes can be rather labile (Wilson and Hodges 1992). This lack of consistency is likely to occur when individuals construct evaluations afresh on the delayed-response occasion. According to this constructivist viewpoint, responses to attitudinal ques- 
tions are based on a search for relevant information in memory (Feldman and Lynch 1988; Tourangeau and Rasinski 1988).

In contrast, delayed attitudes tend to be consistent with initial attitudes when initial attitudes are accessible in memory (Allport 1935; Lingle and Ostrom 1979; Petty and Cacioppo 1981). This model is based on the premise that attitudes are stored in memory as evaluative responses toward objects. When responding to an attitude scale, the individual retrieves this stored evaluative response from memory and reports it on the response scale provided; it is not functional for people to have to construct their attitudes toward the same object anew on every fresh encounter (Fazio 1995). Thus, according to this retrieval perspective, respondents attempt to recall an earlier attitude and use it in order to form their current response (Lavine et al. 1998).

\section{The Effect of Response Mode on Attitude Retrieval versus Construction}

For delayed attitudes, there is, therefore, a trade-off between constructing attitudes anew based on relevant information retrieved from memory and retrieving and reporting attitudes that had been formed earlier. We propose that a match between the response mode of an initial and a delayed attitude will facilitate the accessibility of the initial attitude. There are several related theoretical models that provide a basis for predicting this outcome. These are encoding specificity (Tulving and Thomson 1973), transfer-appropriate processing (Morris, Bransford, and Franks 1977), and repetition of operations (Kolers and Roediger 1984).

Encoding specificity relates to the idea that memory performance tends to be maximized when participants are presented with the same stimulus situation at learning and at the time of test. Originally, this idea related to the conceptual interaction of cues at learning, such that the retrieval cue "a card game" for the target word bridge was less successful when bridge was encoded in the context of "an engineering structure." However, subsequent research has shown that noninteractive or incidental contextual cues at learning can successfully act as retrieval cues, such as being in the same room at test as during learning (Marsh, Edelman, and Bower 2001).

In contrast, transfer-appropriate processing is not concerned with the stimulus context but with the intentional act of the encoder. The original idea concerned perceptual and semantic operations, such that memory for the word eagle is enhanced by the overlap between memory instructions (e.g., "look for words that rhymed with those that were studied") and the task at learning (e.g., "_ is a bird" vs. "_ rhymes with legal"). Recent research has found that transfer-appropriate processing also extends to specific motor actions. For example, after typing a digit string at study, recognition memory was better when the to-be-recognized digit strings were retyped at test compared to when they were simply judged as "old" and "new" in the presence of the keypad (Fendrich, Healy, and Bourne 1991). Moreover, this facilitating effect was attenuated when motoric enact- ment with one hand at study was repeated with the other hand at test, rather than with the same hand on both occasions (Engelkamp et al. 1994).

Finally, repetition of operations was originally concerned with a procedural replication - analytical or cognitive operations-between learning and memory test (e.g., reading text upside down) that was independent of the information content (Kolers and Roediger 1984). Subsequent research has shown the degree of learning to represent a continuum of increasing similarity between encoding and retrieval operations. For example, Glisky and Rabinowitz (1985) included conditions in which words were generated or read at learning and generated or read just prior to answering yes or no on a recognition test. When a word was generated at learning (e.g., MATERIAL from MAT_RI_L), memory was highest when it was again generated from the same word fragment at test, intermediate when it was generated from a different word fragment at test (M_TER_AL), and lowest when it was only read at test.

While there are conceptual distinctions among the original formulations of encoding specificity, transfer-appropriate processing, and repetition of operations, the research streams have been converging and are often treated as part of the same underlying mnemonic mechanism: greater overlap between procedural and representational factors during encoding and memory retrieval will enhance episodic memory for an event.

This idea is particularly relevant with respect to differences in speaking versus typing (for a review, see Garman [1990]). There are obvious differences in the recruitment of appropriate motor systems: the complex muscle movement system for speech-including programs for controlling laryngeal vibration, airflow, and articulation-differ from the arm, hand, and finger movements required for typing. There are also production-planning differences in auxiliary cognitive systems for spoken and typed responses, including the creation or retrieval of different kinds of linguistic structures in separate phonological and orthographic output lexicons and output buffers. For example, when people write, but not when they speak, graphemic representations must be activated prior to the execution of motor programs for handwriting or typing. There are also differences in the perceptual mechanisms involved, for example, in monitoring the verbal outputs via ear and eye.

Neurological evidence reinforces the notion that the writing system is not simply scaffolded on top of the phonological system. In some patients, centers for speech and writing are completely independent. A patient whose corpus callosum had been severed could name pictures aloud when they were presented to the left hemisphere, but she could not write the corresponding words. In contrast, when they were presented to the right hemisphere, she could write them but she could not read them aloud (Baynes et al. 1998). These neurological differences are especially noteworthy as memory storage for an experience appears to reside in the same neural units that processed the experience when it happened in the first place. Successful retrieval then reac- 
tivates the same mental and neural processes that occurred during encoding. For example, when people performed motor actions during encoding and later recalled the event, the motor cortex was reactivated (Nillson et al. 2000).

It is important to recognize that previous research has examined memory for externally provided information. In contrast, attitudes are internal cognitive events. It is likely that self-generated retrieval cues such as speaking versus typing are even more potent for attitudes than for factual information. For example, recall was improved when participants were in the same mood at retrieval as at encoding (Bower 1981), although this effect is labile and dependent on a number of factors (for a review, see Eich [1995]). Most relevant for present purposes, mood appears to be a more potent retrieval cue for self-generated words at learning (word-fragment completion) than for read items (e.g., Eich and Metcalfe 1989) and when respondents have to rely on internal resources rather than on external retrieval aids, such as during free recall versus cued recall or recognition (e.g., Bower 1981; Eich and Metcalfe 1989). We argue that activating the internal processes necessary for speaking and typing will similarly act as retrieval cues and facilitate the accessibility of the initial attitude when the response mode for the delayed attitude is matched.

Finally, a person's own voice or the font setting on a Web browser may also be integrated as part of the memorial representation of initial attitudes and facilitate access to the attitude when voice and font are reinstated at retrieval. For example, study-to-test changes in modality, font, or voice can result in a decrement in recognition memory (Craik and Kirsner 1974). While consistent with our theorizing, this effect is likely to be minor because factors such as one's own voice are not distinctive memory cues.

To summarize, speaking and typing are differentiated in terms of the cognitive systems and processes involved as well as in their motoric manifestation. Our purpose is not to investigate the specific influence of perceptual, encoding, and motor mechanisms involved. Instead, we propose more generally that a spoken or typed response will be integral with the encoding of an initial attitude. Reinstating the same process for a delayed attitude should serve as a retrieval cue that makes the initial attitude more accessible. When initial attitudes are diagnostic, the greater their accessibility, the greater should be the correspondence between initial and delayed attitudes (Allport 1935; Feldman and Lynch 1988; Lingle and Ostrom 1979; Petty and Cacioppo 1981).

\section{CONSEQUENCES OF ATTITUDE RETRIEVAL FAILURE}

Attitudes are a learned predisposition to respond in a consistently favorable or unfavorable manner with respect to a given object (Fishbein and Ajzen 1975) and serve as an internal state of readiness for response (Allport 1935). Examining attitudes from the viewpoint of encoding specificity, transfer-appropriate processing, and repetition of operations is a significant extension of these literatures. These research streams have been concerned primarily with episodic memory for facts. When facts are not accessible in memory, a person may engage in active retrieval strategies, including a search for relevant retrieval cues. These cues can help retrieve the fact in question, but they do not alter it. It is either retrieved or it is not. In the case of a failure to retrieve an attitude for repeated expression, a retrieval strategy is based on retrieving object-specific and other relevant information (Feldman and Lynch 1988; Tourangeau and Rasinsky 1988). This information has the potential to alter the judgment because it is used as an input into constructing the delayed attitude. If the information retrieved differs from that available during the formation of the initial attitude, it is likely that the initial and delayed attitudes will differ in valence.

Experiment 1 tests the hypothesis that a match between the mode of expressing an initial attitude and the mode of response for a delayed attitude will lead to greater consistency. Experiment 2 extends this idea to a delayed-choice behavior. Experiment 3 then tests the underlying process explanation: that a repeated attitude is more likely to be based on the retrieval of the initial attitude when expressed in the same response mode, whereas a delayed attitude is more likely to be based on the process of attitude construction when response modes differ.

\section{EXPERIMENT 1: ATTITUDE CONSISTENCY}

\section{Method}

Design and Procedure. Ninety-three American students participated in the 2 (presentation modality: auditory or visual) $\times 2$ (initial attitude: spoken or typed) $\times 2$ (delayed attitude: spoken or typed) between-subjects experiment. Participants were individually engaged in a series of tasks for which they sat alone in a soundproof room in front of a computer. At the beginning of the experiment, participants were exposed to information about three Canadian candy bars that were not available in the United States. For example, the description of one of the candy bars was

Mr. Big candy bar has moderate availability in Canada. In 1985-86 the brand needed a new strategy, and the idea was quite simple: make Mr. Big BIG! In 1987, the bar was upsized, the pack was flashed with "now gigantic" and the catchy line, "When you're this big, they call you Mister" was used. Mr. Big was repositioned to target teenagers. The result was spectacular; and sales almost doubled. The success of Mr. Big to date is largely due to its strong performance against the teen target group. Teens and preteens consume $31 \%$ of all chocolate bars. However, they consume $52 \%$ of all Mr. Big bars. Teens are very impressionable. To stay foremost in their minds, a chocolate bar must be advertised and be involved in consumer promotions. In advertising, Mr. Big is positioned on more than its size alone. Teens today strive to be fun, hip, and cool . . . so Mr. Big must be portrayed 
as fun, hip and cool as well. Mr. Big has a 100 day shelf life.

Half of the participants read this information, and half of the participants heard this information via speakers. Immediately after exposure, participants judged each of the candy bars on two attitudinal scales. They either heard the instructions and scale items and responded by speaking their response into a microphone, or they read this information and responded by typing their response. Next, they participated in filler tasks requiring both speaking and typing for $45 \mathrm{~min}$. They were then unexpectedly asked to again express their attitudes about the candy bars on two different scales. Half of the participants were asked to respond to these delayed attitude scales in the same mode as they had expressed initial attitudes, and half of the participants responded in a different mode. At the end of the experiment, participants were asked about specific knowledge of the candy bars, provided demographic information, and debriefed.

Dependent Measures. For initial attitudes toward each brand of candy bar, participants either spoke or typed their response to the following questions: "Do you think that is very good, pretty good, neither good nor bad, pretty bad, or very bad?" and "Do you think that _ is a candy bar you would like very much, like somewhat, neither like nor dislike, dislike somewhat, dislike very much?" For each response, participants had to type or speak the entire statement that best represented their attitude. For example, they would respond, "I think that Mr. Big is pretty good." For the delayed attitudes, they were unexpectedly queried again with the questions: "Do you think of _ as highly favorable, mildly favorable, neither favorable nor unfavorable, mildly unfavorable, or highly unfavorable?" and "Do you think that - is totally great, fairly great, neither great nor awful, fairly awful, totally awful?" The same questions were asked for each of the three different candy bars to reduce memory for the specific response on a scale. Attitude inconsistency was calculated as the mean absolute difference between the initial and the delayed attitude across the three candy bars.

\section{Results}

A repeated-measures ANOVA was performed on the factors presentation modality, response mode for the initial attitude, and response mode for the delayed attitude. The only significant effect was the interaction between the mode of response of the first and the second attitude $(F(1,85)=$ $36.62, p<.0001)$. This interaction effect can be interpreted in a straightforward manner: attitudes were less consistent when the mode of response differed between the initial and delayed attitudes (mean absolute difference between initial and delayed attitude: $M=.69$ for spoken-typed; $M=.73$ for typed-spoken) than when they were matched $(M=.21$ for spoken-spoken; $M=.30$ for typed-typed; $F(1,85)=$ $37.60, p<.0001)$. Put differently, in terms of correlations, the initial and delayed attitudes correlated less strongly when response modes differed $(\rho=.25, p<.05$, for spokentyped; $\rho=.21, p<.10$, for typed-spoken) than when they matched $(\rho=.89, p<.0001$, for spoken-spoken; $\rho=.85$, $p<.0001$, for typed-typed). The means are summarized in table 1.

\section{Discussion}

The results of experiment 1 found support for the hypothesis that matching the mode of response results in more consistent attitudes. The main purpose of the second experiment was to eliminate the alternative explanation that, although the actual scales differed, consistency-seeking participants may have been more successful at recalling their relative response to the initial attitude when the response modes matched (Lingle and Ostrom 1979). In a strict sense, the retrieval model of attitude response refers not only to the initial attitude being retrieved from memory but also to that being reported as the delayed attitude. For instance, Lavine et al. (1998) define the retrieval process as consisting of "retrieving the stored evaluation from long-term memory and mapping it onto a given response scale" (359). Thus, retrieval ensures consistency because the delayed attitude that is reported is essentially the same as the initial attitude. However, to better isolate attitudes as internal readiness for response (Allport 1935) from response recall, we selected a verbally expressed choice behavior.

\section{EXPERIMENT 2: ATTITUDE-CHOICE CONSISTENCY}

\section{Method}

Design and Procedure. Ninety-eight American students participated in the 2 (presentation modality: auditory or visual) x 2 (initial attitude: spoken or typed) x 2 (delayed choice: spoken or typed) between-subjects experiment. The procedure was similar to that in experiment 1 , except that participants were exposed to information about only one of the candy bars (Mr. Big). Moreover, the delay between the initial attitude assessment and the choice measure was $1 \mathrm{hr}$. and $15 \mathrm{~min}$. longer than in experiment 1.

Dependent Measures. Initial attitudes were assessed in the same way as in experiment 1. For the unexpected

TABLE 1

ATTITUDE CONSISTENCY IN EXPERIMENT 1

\begin{tabular}{lll}
\hline \hline & \multicolumn{2}{c}{ Delayed attitude } \\
\cline { 2 - 3 } Initial attitude & Spoken & Typed \\
\hline $\begin{array}{l}\text { Spoken: } \\
\quad \text { Mean absolute difference (SD) }\end{array}$ & $.21(.31)$ & $.69(.56)$ \\
$\quad$ Correlation & .89 & .25 \\
$\begin{array}{l}\text { Typed: } \\
\text { Mean absolute difference (SD) }\end{array}$ & $.73(.62)$ & $.30(.36)$ \\
$\quad$ Correlation & .21 & .85 \\
\hline
\end{tabular}


delayed-choice measure, participants read or heard, "Mr. Big is expected to be introduced to the U.S. market sometime in the next year. Would you like to receive an email that informs you when and where it becomes available?" In the typed-response mode they then read, "Please indicate your response by clicking the appropriate button below, and entering your email in the text box if you click yes." In the spoken-response mode they heard, "Please indicate your response by saying yes or no. Please also say and spell your email address if you responded yes."

\section{Results}

The dependent measure was a verbal-choice behavior. A logistic regression was performed with four factors: presentation modality of the product information, the mode of response used for the initial attitudes, the mode of response used for the delayed choice, and the average initial attitude score. There were several significant effects. First, initial attitudes were predictive of the choice to receive more information on the product (Wald's $\chi^{2}=7.92, p<.005$ ). Second, parallel to experiment 1 , there was a significant twoway interaction effect between the mode of response for the initial attitude and the mode of response for the choice behavior (Wald's $\chi^{2}=6.75, p<.01$ ). Third, qualifying these effects, there was a three-way interaction among the initial attitude, the mode of response of the first attitude, and the mode of response of the choice (Wald's $\chi^{2}=6.61, p=$ $.01)$.

This pattern of results can be interpreted in a straightforward manner when examining the correlation between the initial attitudes and the choice to receive more information about the brand. The correlations show that the initial attitudes were predictive of the choice behavior in the matched conditions when both the initial attitude and the delayed choice were spoken $(\rho=.55, p<.005)$ and when both the initial attitude and the delayed choice were typed $(\rho=.40, p<.05)$. In contrast, initial attitudes were not predictive of delayed choices in the mismatched conditions when the initial attitude was spoken and the delayed choice was typed $(\rho=-.04, p>.85)$ and when the initial attitude was typed and the delayed choice was spoken $(\rho=.16$, $p>.50)$.

\section{Discussion}

The results of experiment 2 showed that attitudes were more predictive of choice when this was expressed in the same rather than in a different response mode. This suggests that the attitude-consistency results of experiment 1 were not epiphenomenal: the use of a binary choice behavior eliminates the possibility that participants are simply recalling an earlier response and mapping it onto the new scale.

The primary purpose of experiment 3 was to test the underlying process explanation behind the effect that initial attitudes are more likely to be retrieved when delayed attitudes are expressed in the same response mode and that delayed attitudes are more likely to be constructed when response modes differ. When there is a failure to retrieve the initial attitude, respondents are likely to engage in a search for relevant information, including the original information describing the attitude object (Feldman and Lynch 1988; Tourangeau and Rasinsky 1988). When an initial attitude is readily available, there is no need to engage in this type of search behavior. Because the original product information is reactivated during attitude construction, this should result in better recall memory for this information at a later point in time (Lynch, Marmorstein, and Weigold 1988). As an indicator of attitude construction, recall memory for the product information at a point in time after the expression of the delayed attitude should, therefore, be greater when the delayed attitude is expressed in a different rather than the same response mode as the initial stimulusbased attitude.

In order to establish the accessibility of the initial attitude, we employ attitude confidence or "certainty [which] can be considered as an indirect measure of accessibility" (Holland, Verplanken, and Van Knippenberg 2003, 600). Attitude confidence has been found to be significantly correlated with attitude accessibility as measured by response times (e.g., Berger and Mitchell 1989). The causal explanation for their relationship is that the confidence with which an attitude is held is inferred from the ease with which the attitude comes to mind (Holland et al. 2003). We did not use response times as a measure of accessibility because a meaningful use of response times requires participants to respond as quickly as possible (Fazio 1995). A speeded response would interfere with the process of attitude construction, a cognitive process experiment 3 was designed to measure in addition to attitude retrieval.

Finally, we also examined a nonverbal-choice behavior sometime after the delayed attitudes were assessed. When a delayed attitude reactivates the initial attitude, this should enhance the accessibility of the underlying attitude and inflate attitude-behavior correspondence (Kardes, Allen, and Pontes 1993). This is an interesting theoretical notion because it suggests that the intervening act of measuring a delayed attitude can have a profound and perhaps residual effect on the initial attitude: the act of measuring the attitude changes it.

\section{EXPERIMENT 3: ATTITUDE RETRIEVAL VERSUS CONSTRUCTION}

\section{Method}

The procedure of experiment 3 closely followed that of experiment 1, with the following exceptions. Because the modality of presenting the product information (auditory or visual) had no effect in the previous two experiments, the product information about the three Canadian candy bars was always presented visually alongside an image of the wrapped candy bar. Moreover, because the type of matching (spoken-spoken or typed-typed) and mismatching (spokentyped or typed-spoken) had no effect in the previous two experiments, we employed only one matching (spoken- 
spoken) and one mismatching (typed-spoken) condition for purposes of economy. In addition, we assessed participants' confidence in their initial and delayed attitude ratings in order to obtain a measure for each respondent's change in confidence across the two measurement occasions. We used the same standard confidence question immediately after the initial and delayed attitudes were assessed ("How confident are you about your attitudes toward the __ candy bar?" where $1=$ not at all confident to $7=$ extremely confident). Confidence differences between the initial and delayed attitudes were averaged across candy bars.

Unlike in experiment 1, the delay between the initial attitude at time 1 and the delayed attitude at time 2 was only $20 \mathrm{~min}$. This was done in order to increase the likelihood that the product information presented at time 1 was retrievable at time 2 . On the one hand, this reduces the likelihood that initial and delayed attitudes will differ even when delayed attitudes are constructed: the more product information participants recall, the more their delayed attitude will be consistent with the initial attitude (which may itself be more retrievable under a shorter delay). On the other hand, the main purpose of this experiment was to examine underlying cognitive processes and not to maximize differences in attitude consistency.

Experiment 3 also included an additional experimental block at time 3 . This occurred $1 \mathrm{hr}$. after the delayed attitude assessment, a period of time during which participants were engaged in an unrelated experiment. Participants were first told that they should choose one of the three candy bars as an additional thank you for participation (in reality they received a similar U.S. candy bar). They did so by clicking a button next to one of the images of the three candy bars. Unlike in experiment 2, where the choice behavior was a verbal choice, this task was designed to assess the relative predictive validity of the initial versus the delayed attitudes for a nonverbal behavior. Because the clicking behavior is a manual response this should, if at all, favor the typed initial response in the mismatched condition (typed-spoken). In other words, the manipulation is conservative with respect to our expectation that attitudes in the matched condition (spoken-spoken) will be more predictive of choice.

After the choice task, we assessed spoken recall memory. For this purpose, each of the candy bar names was provided in the order that they were originally presented in, and participants were given no time limit to recall the information presented about each. The original information had been presented as bullet points, and there were seven information units for each candy bar. A coder who was blind to the experimental conditions was instructed to count an item as correct when it contained the gist of the original informational unit of a particular candy bar. Because it is easy to generate reasonable guesses about candy bars that were not part of any of the candy bars (e.g., "contains nuts") false positives were subtracted from participants' correct recall scores. Recall scores were summed across the three candy bars.

At the end of the experiment, participants were asked about specific knowledge of the candy bars, provided demographic information, and were debriefed. Eighty-three American students provided complete responses. Two observations were removed from the analysis because their change in attitudes was more than 2.5 standard deviations removed from the mean (4.4 and 5.8 SD), a standard cutoff for outliers.

\section{Results and Discussion}

Delayed attitudes were more consistent with initial attitudes in the matched $(M=.31)$ than in the mismatched response-mode condition $(M=.42 ; t(81)=-1.95, p=$ $.05)$. The difference between the matched and mismatched conditions is smaller than it was in experiment 1, primarily because the difference between the initial and delayed attitudes in the mismatched condition was smaller. This is to be expected, because a shorter delay increases the amount of relevant information that participants can retrieve when they construct attitudes as well as the accessibility of the initial attitude.

The confidence and recall data indicate that there was a relative shift between attitude retrieval (smaller loss of confidence) in the matched response-mode condition and attitude construction (higher recall) in the mismatched response-mode condition. There was a smaller loss of confidence in the matched $(M=-.00)$ than the mismatched response-mode condition $(M=-.47 ; t(81)=3.02, p<$ $.005)$. In contrast, recall memory was higher in the mismatched $(M=6.7)$ than the matched response-mode condition $(M=5.1 ; t(81)=-2.14, p<.05)$.

We examined the degree to which initial and delayed attitudes predicted the choice of candy bars (hit rates) using multinomial logit models. Hit rates in the mismatched response-mode condition were higher for the delayed attitudes $(M=74.5 \%$; log likelihood $[L L]=-31.0)$ than the initial attitudes $(M=59.6 \% ; L L=-34.3)$. In contrast, hit rates in the matched response-mode condition were similar for the delayed $(M=73.7 \% ; L L=-23.0)$ and the initial $(M=71.1 \% ; L L=-27.2)$ attitudes. Nested tests indicate that the delayed attitudes were more predictive of choice in both conditions and that the initial attitudes do not improve model fit. This signals that the more distal attitude does not add explanatory power to the more proximal attitude. To test for significant differences across conditions, we compared the initial and delayed attitudes by interacting the two attitude dummies (and brand dummies) with condition. This analysis showed that initial attitudes were more predictive in the matched than the mismatched condition $(p<.05)$ but that delayed attitudes were equally predictive of choice in both conditions $(p>.80)$.

\section{GENERAL DISCUSSION}

This article applies and extends a procedural view-that memory representations cannot be divorced from the procedures used to acquire them-to the domain of expressed attitudes. Previous "research on learning and memory has 
preserved a distinction between motor processes and processes based on language or 'thought,' usually with a pejorative implication for the former" (Kolers and Roediger 1984, 428-29). Our findings show that differences between speaking and typing are not only mechanical but mediate intellective processes.

Kardes (1986) has shown that initial stimulus-based judgments influence subsequent global memory-based judgments, and Kardes et al. (1993) suggested that assessing an attitude can change it. We demonstrate that these relationships are critically influenced by whether the initial and delayed attitudes are expressed using the same response mode. We propose that the cognitive and motor actions involved in expressing the initial attitude become an integral part of its memory trace. Activating similar processes during a subsequent attitude expression made the initial attitude more accessible and consistent. Mismatching the mode of response resulted in a higher likelihood of constructing delayed attitudes based on the retrieval of attribute information, as indicated by a higher level of attribute recall at a later point in time.

Mismatching versus matching the response mode not only affected attitude valence to a greater degree but also decreased confidence. Attitudes held with less confidence are less predictive of future choices (Fazio 1995). In our research, the delayed attitudes were equally predictive in the matched and mismatched conditions, despite differences in confidence. This is likely because participants were forced to choose between the candy bars and attitude confidence was not a differentiator between choice options in either condition. If the choice set had included brands for which attitude confidence varied, this may have influenced brand choice.

\section{Alternative Accounts}

Three experiments demonstrated that mismatching the mode of expressing an initial and a delayed attitude or verbal behavior results in greater inconsistency between the two occasions. A possible alternative explanation that is unrelated to the notion of attitude retrieval and construction is that scale usage may have differed between response modes. For example, previous research on response-order effects has shown that face-to-face or telephone interviews can result in a different response bias than self-administered questionnaires (Schwarz et al. 1991). Cross-matching response modes would then artificially create absolute differences between the initial and delayed attitudes.

A response-order effect would be demonstrated empirically by a differential bias on spoken and typed responses. Table 2 summarizes the raw means for responses to attitude and confidence scales across the three experiments. These speak against the possibility of a response-order effect. The five possible direct (columnar) comparisons did not show a differential bias for spoken and typed responses in a metaanalysis $(Z<1, \mathrm{NS})$. This lack of a response-order effect may seem surprising, but response-order effects critically depend on the nature of the question. They are typically
TABLE 2

RAW CELL MEANS (SD)

\begin{tabular}{|c|c|c|}
\hline \multirow[b]{2}{*}{ Measurement occasion } & \multicolumn{2}{|c|}{ Response mode } \\
\hline & Spoken & Typed \\
\hline \multicolumn{3}{|l|}{ Experiment 1 : } \\
\hline Initial attitude & $3.70 \quad(.73)$ & $3.71 \quad(.80)$ \\
\hline Delayed attitude & $3.70 \quad(.76)$ & $3.67 \quad(.77)$ \\
\hline \multicolumn{3}{|l|}{ Experiment 2: } \\
\hline Initial attitude & $3.64 \quad(.59)$ & $3.57 \quad(.85)$ \\
\hline \multicolumn{3}{|l|}{ Experiment 3: } \\
\hline Initial attitude & $2.61 \quad(.46)$ & $2.27 \quad(.47)$ \\
\hline $\begin{array}{l}\text { Delayed attitude (spoken-spoken } \\
\text { condition) }\end{array}$ & $2.61 \quad(.57)$ & \\
\hline $\begin{array}{l}\text { Delayed attitude (typed-spoken } \\
\text { condition) }\end{array}$ & $2.52(.64)$ & \\
\hline Initial confidence & $4.14(1.06)$ & $4.43(1.08)$ \\
\hline $\begin{array}{l}\text { Delayed confidence (spoken-spoken } \\
\text { condition) }\end{array}$ & $4.14(1.15)$ & \\
\hline $\begin{array}{l}\text { Delayed confidence (typed-spoken } \\
\text { condition) }\end{array}$ & $3.96 \quad(.79)$ & \\
\hline
\end{tabular}

obtained when respondents are asked to select from a set of qualitatively different opinions the one that most closely represents their own. Differences in the opportunity to elaborate on items presented in different parts of the list and the difficulty of keeping items in mind without visual help can result in recency effects for aurally presented response sets and primacy effects for self-administered questionnaires (Schwarz et al. 1991). Response-order effects should not be expected for unidimensional attitude scales, especially not when the scale is repeated across stimulus alternatives as in the current setting (Norbert Schwarz, personal communication).

A different alternative explanation is based on the perceived ease with which respondents are able to retrieve delayed attitudes from memory, what we discussed as the causal driver of attitude confidence (Holland et al. 2003). Recent research on the mere-measurement effect suggests that the sensation of greater ease with which accessible attitudes come to mind can be interpreted by respondents as a predisposition toward the attitude object, thereby resulting in a more positive evaluation (Janiszewski and Chandon 2007). We do not find empirical evidence for this type of effect: attitudes in the matched $(M=3.74)$ and mismatched conditions $(M=3.64)$ did not differ significantly in experiment $1(t(277)=1.12, p>.25)$ or in experiment 3 (see table $2 ; t(81)=.66, p>.50)$. This suggests that the greater attitude inconsistency in the mismatching conditions was not caused by a directional attitudinal bias.

Finally, we found that the amodal choice behavior in the mismatched condition was better predicted by the more proximal delayed attitude (60 min. prior) than the initial attitude (80 min. prior). We interpret this effect as evidence for the delayed attitude having changed the initial attitude (Kardes et al. 1993). It is possible, however, that the initial attitude was not overridden but continued to coexist in memory, creating a situation of dual attitudes (Wilson, Lindsey, 
and Schooler 2000). We do not believe that the conditions of our experiments lend themselves to the formation of dual attitudes or that our results could have been influenced by the existence of dual attitudes (i.e., they tend to surface in implicit tasks). However, it remains an interesting question, whether the related concept of attitude ambivalence (Kaplan 1972) can surface across response modes, because spoken and typed responses may tap into different aspects of representation. Under certain conditions individuals may, therefore, evaluate an object positively in one response mode and negatively in another response mode.

\section{Summary}

While attitudes and behaviors have commonly been expressed orally, the rise of the Internet and of text messaging on cellular telephones is associated with a sharp increase in typed expressions of beliefs, opinions, and behaviors. These different response modes do not simply express an attitude but change its representation. This not only has implications for everyday behaviors but also for marketing researchers and opinion pollsters. For example, if researchers are interested in how attitudes decay over time, then ensuring consistency in the response mode is critical. Similarly, when attitudes are measured in an attempt to predict future attitudes or future behavior, our results suggest researchers should strive to match the mode of initial attitude expression with that of subsequent attitude measurement or behavior. Our results do not, however, imply that it is always optimal to rely on the same response mode to repeatedly assess attitudes. Marketing interventions are often designed to change attitudes. As such, a marketing intervention between an initial and delayed attitude should have a stronger impact on attitude change when response modes are mismatched due to the increased likelihood that the response is constructed and will incorporate the intervention.

To our knowledge, our findings are the first to show that verbal production mechanisms affect attitude retrieval versus construction. The legacy of behaviorism may be one culprit for this negligence. Watson's original conception of cognition put forth the somewhat absurd notion that "thought processes are really motor habits in the larynx" $(1913,174)$, the prototype for what was dubbed the mind-in-the-mouth assumption (Bock 1996). The counterreaction to behaviorist ideas only compounded the problem and led to inattention in how people control their responses within experimental tasks (Bock and Griffin 2000). We hope that our findings may spark renewed interest in how production mechanisms affect intellective processes.

\section{REFERENCES}

Allport, Gordon W. (1935), “Attitudes," in Handbook of Social Psychology, ed. C. Murchison, Worcester, MA: Clark University Press, 789-844.

Baynes, Kathleen, James C. Eliassen, Helmi L. Lutsep, and Michael S. Gazzaniga (1998), "Modular Organization of Cog- nitive Systems Masked by Interhemispheric Integration," Science, 280 (May), 902-5.

Berger, Ida E. and Andrew A. Mitchell (1989), "The Effect of Advertising on Attitude Accessibility, Attitude Confidence, and the Attitude-Behavior Relationship," Journal of Consumer Research, 16 (December), 269-79.

Bock, J. Kathryn (1996), "Language Production: Methods and Methodologies," Psychonomic Bulletin and Review, 3 (December), 395-421.

Bock, J. Kathryn and Zenzi M. Griffin (2000), "Producing Words: How Mind Meets Mouth," in Aspects of Language Production, ed. Linda R. Wheeldon, East Sussex: Psychology, 7-47.

Bower, Gordon H. (1981), "Mood and Memory," American Psychologist, 36 (February), 129-48.

Cook, Thomas D. and Brian R. Flay (1978), "The Temporal Persistence of Experimentally Induced Attitude Change: An Evaluative Review," in Advances in Experimental Social Psychology, ed. L. Berkowitz, New York: Academic Press, 1-57.

Craik, Fergus I. M. and Kim Kirsner (1974), "The Effect of Speaker's Voice on Word Recognition," Quarterly Journal of Experimental Psychology, 26 (2), 274-84.

Eich, Eric (1995), "Searching for Mood Dependent Memory," Psychological Science, 6 (March), 67-75.

Eich, Eric and Janet Metcalfe (1989), "Mood Dependent Memory for Internal versus External Events," Journal of Experimental Psychology: Learning, Memory, and Cognition, 15 (May), 443-55.

Engelkamp, Johannes, Hubert D. Zimmer, Gilbert Mohr, and Odmar Sellen (1994), "Memory for Self-Performed Tasks: SelfPerforming during Recognition," Memory and Cognition, 22 (January), 34-39.

Fazio, Russel H. (1995), “Attitudes as Object-Evaluation Associations: Determinants, Consequences, and Correlates of Attitude Accessibility," in Attitude Strength: Antecedents and Consequences, ed. R. E. Petty and J. A. Krosnick, Mahwah, NJ: Erlbaum, 73-92.

Feldman, Jack M. and John G. Lynch Jr. (1988), "Self-Generated Validity and Other Effects of Measurement on Belief, Attitude, Intention, and Behavior," Journal of Applied Psychology, 73 (August), 421-35.

Fendrich, David W., Alice F. Healy, and Lyle E. Bourne Jr. (1991), "Long-Term Memory Repetition Effects for Motoric and Perceptual Procedures," Journal of Experimental Psychology: Learning, Memory, and Cognition, 17 (January), 137-51.

Fishbein, Martin and Icek Ajzen (1975), Belief, Attitude, Intention, and Behavior: An Introduction to Theory and Behavior, Reading, MA: Addison-Wesley.

Garman, Michael (1990), Psycholinguistics, Cambridge: Cambridge University Press.

Glisky, Elizabeth L. and Jan C. Rabinowitz (1985), "Enhancing the Generation Effect through Repetition of Operations," Journal of Experimental Psychology: Learning, Memory, and Cognition, 11 (April), 193-205.

Holland, Rob W., Bas Verplanken, and Ad van Knippenberg (2003), "From Repetition to Conviction: Attitude Accessibility as a Determinant of Attitude Certainty," Journal of Experimental Social Psychology, 39 (November), 594-601.

Janiszewski, Chris and Elise Chandon (2007), "Transfer Appropriate Processing, Response Fluency, and the Mere Measurement Effect," Journal of Marketing Research, 43 (February), forthcoming.

Kaplan, Kalman J. (1972), "On the Ambivalence-Indifference Problem in Attitude Theory and Measurement: A Suggested 
Modification of the Semantic Differential Technique," Psychological Bulletin, 77 (May), 361-72.

Kardes, Frank R. (1986), "Effects of Initial Product Judgments on Subsequent Memory-Based Judgments," Journal of Consumer Research, 13 (June), 1-11.

Kardes, Frank R., Chris T. Allen, and Manuel J. Pontes (1993), "Effects of Multiple Measurement Operations on Consumer Judgment: Measurement Reliability or Reactivity?" Advances in Consumer Research, 20, 280-83.

Kolers, Paul A. and Henry L. Roediger III (1984), "Procedures of Mind," Journal of Verbal Learning and Verbal Behavior, 23 (August), 425-49.

Lavine, Howard, Joseph W. Huff, Stephen H. Wagner, and Donna Sweeney (1998), "The Moderating Influence of Attitude Strength on the Susceptibility to Context Effects in Attitude Surveys," Journal of Personality and Social Psychology, 75 (August), 359-73.

Lingle, John and Thomas Ostrom (1979), "Retrieval Selectivity in Memory-Based Impression Judgments," Journal of Personality and Social Psychology, 37 (February), 180-94.

Lynch, John G., Jr., Howard Marmorstein, and Michael F. Weigold (1988), "Choices from Sets Including Remembered Brands: Use of Recalled Attributes and Prior Overall Evaluations," Journal of Consumer Research, 15 (September), 169-84.

Marsh, Elizabeth J., Gabriel Edelman, and Gordon H. Bower (2001), "Demonstrations of a Generation Effect in Context Memory," Memory and Cognition, 29 (September), 798-805.

Morris, C. Donald, John D. Bransford, and Jeffrey J. Franks (1977), "Levels of Processing versus Transfer Appropriate Process- ing," Journal of Verbal Learning and Verbal Behavior, 16 (October), 519-33.

Nillson, L., L. Nyberg, T. Klingberg, C. Aberg, J. Persson, and P. E. Roland (2000), "Activity in Motor Areas while Remembering Action Events," Neuroport: For Rapid Communication of Neuroscience Research, 11, 2199-2201.

Petty, Richard E. and John T. Cacioppo (1981), Attitudes and Persuasion: Classic and Contemporary Approaches, Dubuque, IA: Brown.

Schwarz, Norbert, Fritz Strack, Hans-J. Hippler, and George Bishop (1991), "The Impact of Administration Mode on Response Effects in Survey Measurement," Applied Cognitive Psychology, 5 (May-June), 193-212.

Tourangeau, Roger and Kenneth A. Rasinski (1988), "Cognitive Processes Underlying Context Effects in Attitude Measurement," Psychological Bulletin, 103 (May), 299-314.

Tulving, Endel and Donald M. Thomson (1973), "Encoding Specificity and Retrieval Processes in Episodic Memory," Psychological Review, 80 (5), 359-80.

Watson, John B. (1913), "Psychology as the Behaviorist Views It," Psychological Review, 20, 158-77.

Wilson, Timothy D. and Sara D. Hodges (1992), "Attitudes as Temporary Constructions," in The Construction of Social Judgments, ed. Leonard L. Martin and Abraham Tesser, Hillsdale, NJ: Erlbaum, 37-65.

Wilson, Timothy D., Samuel Lindsey, and Tonya Y. Schooler (2000), "A Model of Dual Attitudes," Psychological Review, 107 (January), 101-26. 